\title{
Scattering mechanisms in high-mobility strained Ge channels
}

\author{
B. Rössner \\ Laboratorium für Festkörperphysik, ETH Zürich, CH-8093 Zürich, Switzerland \\ D. Chrastina, ${ }^{a}$ G. Isella, and H. von Känel \\ INFM and L-NESS Dipartimento di Fisica, Politecnico di Milano, Polo Regionale di Como, Via Anzani 52, \\ I-22100 Como, Italy
}

(Received 29 December 2003; accepted 20 February 2004)

\begin{abstract}
We report on the low-temperature mobility in remotely doped $p$-type strained Ge layers on relaxed $\mathrm{Si}_{0.3} \mathrm{Ge}_{0.7}$ virtual substrates, grown by low-energy plasma-enhanced chemical vapor deposition. $\mathrm{A}$ maximum mobility of $120000 \mathrm{~cm}^{2} \mathrm{~V}^{-1} \mathrm{~s}^{-1}$ has been reached at $2 \mathrm{~K}$, at a carrier sheet density of $8.5 \times 10^{11} \mathrm{~cm}^{-2}$. Analysis of the mobility and Dingle ratio $\tau / \tau_{q}$ as a function of sheet density suggests that remote impurity scattering is the limiting factor at low sheet densities, but that interface impurities become more important as the sheet density increases. (C) 2004 American Institute of Physics. [DOI: 10.1063/1.1707223]
\end{abstract}

The SiGe material system greatly extends the performance and versatility of Si-based semiconductors. In particular, compressively strained Ge channels can show high $p$-type mobilities. A $4.2 \mathrm{~K}$ mobility of $87000 \mathrm{~cm}^{2} \mathrm{~V}^{-1} \mathrm{~s}^{-1}$ (at $6.2 \times 10^{11} \mathrm{~cm}^{-2}$ ) has already been reported ${ }^{1}$ and roomtemperature mobilities of around $3000 \mathrm{~cm}^{2} \mathrm{~V}^{-1} \mathrm{~s}^{-1}$ have been found using mobility spectrum analysis. ${ }^{1-3}$ Some of the physical properties of holes in strained Ge have been studied. ${ }^{4-7}$ Here, we analyze the sheet density dependence of the mobility in high-mobility material.

$p$-type modulation-doped quantum well ( $p$-MODQW) samples were grown by low-energy plasma-enhanced chemical vapor deposition (LEPECVD). ${ }^{8}$ Their basic structure is shown in Fig. 1. (The structure of sample 6016 is detailed in Ref. 1. All others are identical apart from sample 6745, which features a thinner cap.) The virtual substrate (VS) was grown at $5-10 \mathrm{~nm} \mathrm{~s}^{-1}$, using high rate plasma conditions, ${ }^{1}$ at substrate temperatures from $740^{\circ} \mathrm{C}$ falling to $500^{\circ} \mathrm{C}$ with increasing Ge content. The Ge fraction of the VS was increased linearly from zero to 0.7 over $10 \mu \mathrm{m}$, followed by a $2 \mu \mathrm{m} \mathrm{Si}_{0.3} \mathrm{Ge}_{0.7}$ constant composition layer. The active layers were grown at $0.3 \mathrm{~nm} \mathrm{~s}^{-1}$, using a plasma of reduced density, at a substrate temperature of $450{ }^{\circ} \mathrm{C}$. There was no growth interruption between the graded buffer and the active layers. The transition between high and low growth rate plasma conditions takes a few seconds.

For electrical characterization, the samples were cleaved into squares of $3 \times 3$ to $5 \times 5 \mathrm{~mm}^{2}$. The corners were contacted manually using In soldering to Au wire. Contact size ranged from 0.2 to $0.4 \mathrm{~mm}$. For electrical characterization in this van der Pauw geometry, a standard lock-in technique was used with frequencies ranging from 20 to $40 \mathrm{~Hz}$ and a current of $100 \mathrm{nA}$. Heating effects were not detected at this current level.

Directly after soldering, the measured sheet density was generally lower than that measured after letting the sample rest at RT for several hours. At low-temperature, the sheet density was found to be stable over time. We assume that the

a)Electronic mail: daniel@chrastina.net heating during soldering causes modifications of the sample surface, which relax only slowly at RT. This in turn causes charge to be trapped at the surface, which changes the position of the bands relative to the Fermi level and modulates the sheet density. Therefore, by temperature cycling between 300 and $2 \mathrm{~K}$, it was possible to obtain different sheet densities in the same sample.

Samples were measured at temperatures down to $2 \mathrm{~K}$ at fields of up to $4 \mathrm{~T}$. The effective mass $m^{*}$ and Dingle ratio $\alpha=\tau / \tau_{q}$ (where $\tau$ is the transport scattering time and $\tau_{q}$ is the quantum scattering time) were determined from a fit of the amplitude of the Shubnikov-de Haas ( $\mathrm{SdH}$ ) oscillations. ${ }^{9,10}$ To this end, $\mathrm{SdH}$ oscillations of two different van der Pauw contact arrangements were measured sequentially and combined to obtain the sheet resistance.

Additionally, Hall bars were fabricated using standard photolithography and wet chemical etching. Al contact pads were deposited using e-beam evaporation. The Hall bar 6843 was also measured following illumination with a green lightemitting diode. Mobilities and sheet densities were derived from the low-field Hall effect.

Mobility results are shown in Fig. 2. The general trend is that the mobility increases with sheet density. A maximum of $120000 \mathrm{~cm}^{2} \mathrm{~V}^{-1} \mathrm{~s}^{-1}$ was measured in sample 6745 at a

\begin{tabular}{|l|}
\hline $3 \mathrm{~nm} \mathrm{Si}$ \\
\hline $20 \mathrm{~nm} \mathrm{Si} \mathrm{S}_{0.3} \mathrm{Ge}_{0.7}$ \\
\hline $2 \mathrm{p}$-type $(\mathrm{B})$ d-doping spikes \\
\hline $10 \mathrm{~nm} \mathrm{Si} \mathrm{Ge}_{0.7}$ \\
\hline $15 \mathrm{~nm} \mathrm{Ge} \mathrm{QW}$ \\
\hline $50 \mathrm{~nm} \mathrm{Si}{ }_{0.3} \mathrm{Ge}_{0.7}$ \\
\hline $12 \mu \mathrm{m}$ Graded buffer $\mathrm{Si}-\mathrm{Si}_{0.3} \mathrm{Ge}_{0.7}$ \\
\hline $\mathrm{Si}(001)$ n-type substrate \\
\hline
\end{tabular}

FIG. 1. The basic structure. This figure applies directly to devices 6747 , 6777, and 6843. The structure of sample 6016 is detailed in Ref. 1. 6745 features a $\mathrm{Si}_{0.3} \mathrm{Ge}_{0.7}$ cap (above the doping spikes) of only $10 \mathrm{~nm}$. 


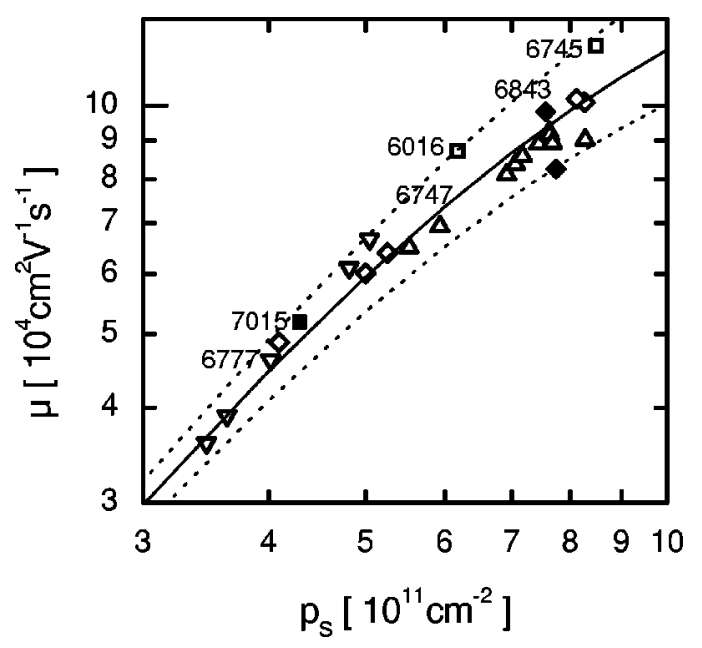

FIG. 2. Mobility at $2 \mathrm{~K}$ for $p$-MODQWs grown by LEPECVD. Different sheet densities were induced in some samples $[6747(\triangle), 6777(\nabla), 6843$ $(\diamond)]$ by exploiting the effects of annealing on the pinning of surface states. All other samples $(\square)$ are labeled individually. Open symbols are results from square samples, filled symbols are results from Hall bars. (The uppermost point for the Hall bar 6843 followed illumination with a green lightemitting diode.) The solid line was calculated using the parameters in Table I (with $n_{i}=1.5 \times 10^{9} \mathrm{~cm}^{-2}$ ). The upper and lower dotted lines were calculated with $n_{i}=1.0$ and $2.0 \times 10^{9} \mathrm{~cm}^{-2}$ instead.

sheet density of $8.5 \times 10^{11} \mathrm{~cm}^{-2}$. Despite the fact that most of the samples were grown with nominally the same structure, there is a spread of sheet densities. This is due to a memory effect of the growth system: impurities are introduced into the VS by redeposition from the chamber walls. If this contamination is strong, then it will show up in the interface impurity scattering rate. Otherwise, it has the effect of weak doping underneath the channel and does not significantly change the scattering parameters. (A term could be incorporated into the effective field, for example, but it would be small compared to the field from the intentional doping.)

The increase of mobility with respect to sheet density is a general signature of impurity scattering, from either interface or remote impurities. Dingle ratios are shown in Fig. 3. Scattering from interface impurities or interface roughness

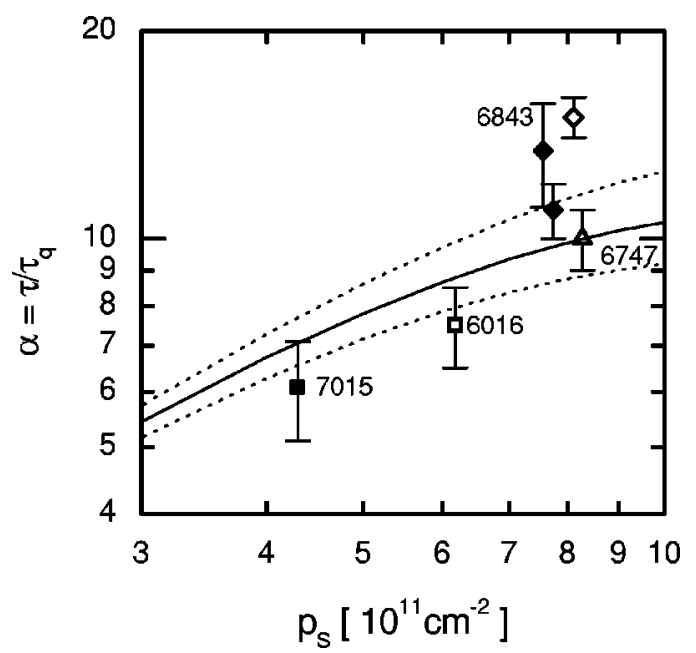

FIG. 3. Dingle ratios at $2 \mathrm{~K}$. Open symbols are results from square samples, filled symbols are results from Hall bars. The solid and dotted lines are calculated as in Fig. 2.
TABLE I. The parameters which give the lines in Figs. 2, 3, and 4. $n_{D}, S$, and $W$ relate to remote impurity scattering and $n_{i}$ relates to local impurity scattering. The values of $\Delta$ and $\Lambda$ can only be estimated since interface roughness scattering is not a strong feature.

\begin{tabular}{cc} 
Remote impurity density $n_{D}$ & $4.5 \times 10^{12} \mathrm{~cm}^{-2}$ \\
Effective setback $S$ & $14 \mathrm{~nm}$ \\
Depletion width $W$ & $22 \mathrm{~nm}$ \\
Interface impurity density $n_{i}$ & $1.5 \pm 0.5 \times 10^{9} \mathrm{~cm}^{-2}$ \\
Roughness height $\Delta$ & $0.1 \mathrm{~nm}$ \\
Roughness length $\Lambda$ & $1.0 \mathrm{~nm}$ \\
\hline
\end{tabular}

gives $\alpha \sim 1$. The fact that our samples show $\alpha \sim 5-10$ suggests the dominance of remote impurity scattering.

In order to shed more light onto the relative contribution of different scattering mechanisms to the measured mobility, we use linear-transport theory neglecting multiple scattering. ${ }^{11} \mathrm{We}$ consider low-temperature scattering from correlated remote impurities, ${ }^{12-14}$ interface or background impurities, ${ }^{15}$ and interface roughness. ${ }^{15}$ The increase of transport-direction effective mass $m^{*}$ with sheet density is taken into account, ${ }^{6}$ but the effective mass in the growth direction $m_{z}$ is fixed at $0.19 m_{e} .{ }^{16}$ We treat screening according to the formulation of Kearney and Horrell ${ }^{15,17}$ since $q_{s}$ $\sim 2 k_{\mathrm{F}}$ at $p_{s} \sim 2 \times 10^{11} \mathrm{~cm}^{-2}$ in strained $p$-type Ge channels. No significant weak localization effects were seen in the low-field magnetoresistance.

The solid lines in Figs. 2 and 3 were calculated using the parameters given in Table I (with $n_{i}=1.5 \times 10^{9} \mathrm{~cm}^{-2}$ ). Remote impurity scattering depends on the remote impurity density $n_{D}$, distance $S$ between ionized impurities and the two-dimensional hole gas, and width $W$ over which dopants are ionized. Interface impurity scattering depends only on the interface impurity density $n_{i}$. Interface roughness scattering depends on the height $\Delta$ and length $\Lambda$ of the (Gaussian) roughness.

The upper and lower dotted lines were calculated with $n_{i}=1.0$ and $2.0 \times 10^{9} \mathrm{~cm}^{-2}$, respectively. All the measured mobilities are explained with the parameters in Table I. Regarding the Dingle ratios in Fig. 3, the form is correct (with an increase of $\alpha$ as $p_{s}$ increases), but the calculations overestimate $\alpha$ slightly at low $p_{s}$. The Dingle ratios measured in sample 6843 are generally underestimated by the calculation. In the case of large Dingle ratios, the measured value may not be reliable due to inhomogeneities in the sheet density. ${ }^{9,12}$

Figure 4 shows the contributions of the various scattering mechanisms. At low sheet densities, $\mu$ is determined almost entirely by remote impurity scattering, with $\mu \propto p_{s}^{1.6}$. If these results were dominated by interface impurities instead, the behavior would be of the form $\mu \propto p_{s}^{0.5}$ and $\alpha$ would be $\sim 1.6$ at most. Dingle ratios of $\sim 10$ require not only that remote impurity scattering be dominant, but also that the depletion width $W$ be relatively large so that the correlation length between dopants is large. ${ }^{13}$ The interface impurity density of $1.5 \times 10^{9} \mathrm{~cm}^{-2}$ given in Table I is almost two orders of magnitude lower than the values obtained for pseudomorphic $\mathrm{Si}_{0.8} \mathrm{Ge}_{0.2}$ channels grown by molecularbeam epitaxy. ${ }^{15,18}$

The interface roughness scattering parameters in the present samples must be somewhat smaller than reported for 


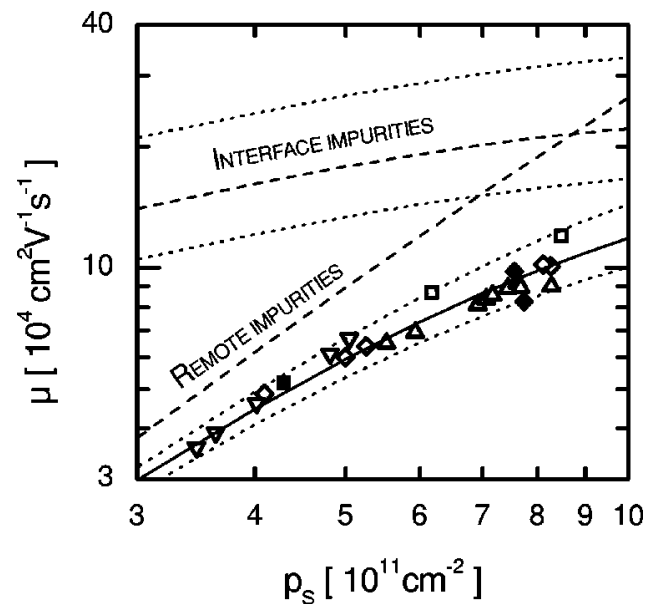

FIG. 4. The contribution of each scattering mechanism to the overall mobility $\mu$. At low sheet densities, remote impurity scattering is dominant; interface impurity scattering becomes more important as sheet density increases. Interface roughness is unimportant, giving a scattering rate corresponding to $\mu>10^{7} \mathrm{~cm}^{2} \mathrm{~V}^{-1} \mathrm{~s}^{-1}$.

the SiGe system, ${ }^{5,18}$ since it does not significantly limit the mobility. At the sheet densities covered in this work, the roughness length $\Lambda$ is much smaller than the Fermi wavelength, so that only the value of the product $\Delta \Lambda$ determines the scattering rate. ${ }^{15}$ Therefore, the value of either parameter alone cannot be estimated: results at higher sheet densities would be necessary, preferably from gated samples for which additional doping is not required.

In summary, mobilities in $p$-type strained Ge QWs have been analyzed using linear-transport theory. The mobility is limited only by scattering from remote impurities at low sheet density. The contribution of interface impurities becomes more important as the sheet density increases. A mo- bility maximum at $2 \mathrm{~K}$ of $120000 \mathrm{~cm}^{2} \mathrm{~V}^{-1} \mathrm{~s}^{-1}$ was measured in sample 6745 at a sheet density of $8.5 \times 10^{11} \mathrm{~cm}^{-2}$. It is to be expected that the effects of interface impurity scattering will eventually be seen in gated samples, in which the sheet density can be further increased.

Financial support from GROWTH Program ECOPRO No. GRD2-2000-30064 is gratefully acknowledged. We would like to thank B. Batlogg for a critical reading of the manuscript.

${ }^{1}$ H. von Känel, M. Kummer, G. Isella, E. Müller, and T. Hackbarth, Appl. Phys. Lett. 80, 2922 (2002).

${ }^{2}$ D. Chrastina, J. P. Hague, and D. R. Leadley, J. Appl. Phys. 94, 6583 (2003).

${ }^{3}$ M. Myronov, P. J. Phillips, T. E. Whall, and E. H. C. Parker, Appl. Phys. Lett. 80, 3557 (2002).

${ }^{4}$ T. Irisawa, M. Myronov, O. A. Mironov, E. H. C. Parker, K. Nakagawa, M. Murata, S. Koh, and Y. Shiraki, Appl. Phys. Lett. 82, 1425 (2003).

${ }^{5}$ S. Madhavi, V. Venkataraman, and Y. H. Xie, J. Appl. Phys. 89, 2497 (2001).

${ }^{6}$ B. Rößner, G. Isella, and H. von Känel, Appl. Phys. Lett. 82, 754 (2003).

${ }^{7}$ R. Winkler, M. Merkler, T. Darnhofer, and U. Rössler, Phys. Rev. B 53, 10858 (1996).

${ }^{8}$ C. Rosenblad, H. R. Deller, A. Dommann, T. Meyer, P. Schroeter, and H. von Känel, J. Vac. Sci. Technol. A 16, 2785 (1998).

${ }^{9}$ P. T. Coleridge, R. Stoner, and R. Fletcher, Phys. Rev. B 39, 1120 (1989).

${ }^{10}$ A. Isihara and L. Smrčka, J. Phys. C 19, 6777 (1986).

${ }^{11}$ T. Ando, A. B. Fowler, and F. Stern, Rev. Mod. Phys. 54, 437 (1982).

${ }^{12}$ P. T. Coleridge, Phys. Rev. B 44, 3793 (1991).

${ }^{13}$ P. J. van Hall, Superlattices Microstruct. 6, 213 (1989).

${ }^{14}$ P. J. van Hall, T. Klaver, and J. H. Wolter, Semicond. Sci. Technol. 3, 120 (1988).

${ }^{15}$ R. J. P. Lander, M. J. Kearney, A. I. Horrell, E. H. C. Parker, P. J. Phillips, and T. E. Whall, Semicond. Sci. Technol. 12, 1064 (1997).

${ }^{16}$ M. V. Fischetti and S. E. Laux, J. Appl. Phys. 80, 2234 (1996).

${ }^{17}$ M. J. Kearney and A. I. Horrell, Semicond. Sci. Technol. 13, 174 (1998).

${ }^{18}$ M. A. Sadeghzadeh, A. I. Horrell, O. A. Mironov, E. H. C. Parker, T. E. Whall, and M. J. Kearney, Appl. Phys. Lett. 76, 2568 (2000). 